\title{
OBSTRUCTION FORMULAS AND ALMOST-COMPLEX MANIFOLDS
}

\author{
ROBERT D. LITTLE
}

ABSTRACT. This paper contains three theorems about almost-complex manifolds. The first theorem states that, under certain conditions, the Euler characteristic of an almost-complex manifold $M^{2 n}$ must be divisible by $(n-1)$ !. This theorem implies that if $M^{2 n}$ is an almost-complex homology sphere, then $n \leq 3$. The next two theorems concern the maximal number of vector fields of an almost-complex manifold which are linearly independent over the complex numbers.

1. Introduction. The purpose of this paper is to prove the three theorems below using obstruction formulas due to Olum [7]. An almost-complex manifold is a closed, connected $C^{\infty}$ manifold $M$ of dimension $2 n$, the tangent bundle of which is isomorphic to the real bundle underlying a complex $n$-plane bundle. By the complex span of such a manifold, we mean the maximal number of vector fields of $M$ which are linearly independent over the complex numbers. We denote the Chern class of $M$ in $H^{2 i}(M ; Z)$ by $c_{i}(M)$ and the Euler characteristic by $\chi(M)$.

Theorem 1. If $M$ is an almost-complex manifold such that $c_{i}(M)=0$, $i<n$, and the torsion coefficients of $H^{2 i}(M ; Z)$ are greater than $i-1$, $3 \leq i \leq n-2$, then $(n-1)$ ! divides $\chi^{(M)}$.

Theorem 2. If $n$ is odd and $n \geq 9$ and $M$ is a simply connected almostcomplex manifold such that $H_{4}(M ; Z)$ bas no 2-torsion and $H_{3}\left(M ; \mathrm{Z}_{p}\right)=0$ for $p=2$ and 3 , then complex $\operatorname{span}(M) \geq 4$ if and only if $c_{i}(M)=0, n-3$ $\leq i \leq n$.

Theorem 3. If $n$ is even, $n \geq 10$ and $n \not \equiv 0(\bmod 8)$ and $M$ is a simply connected almost-complex manifold such that $H_{6}(M ; Z)$ bas no 2-torsion and $H_{i}\left(M ; \mathrm{Z}_{p}\right)=0$ for $i=3,5$ and $p=2,3$, then complex $\operatorname{span}(M) \geq 5$ if and only if $c_{i}(M)=0, n-4 \leq i \leq n$.

Received by the editors April 26, 1974.

AMS (MOS) subject classifications (1970). Primary 55G35, 55G40, 53C15.

Key words and phrases. Almost-complex manifolds, obstructions, lifting problems, vector fields, complex span. 
Theorem 1 is similar to the theorem of Serre and Borel [2, p. 434] which says that if $M$ is an almost-complex manifold with $c_{i}(M)=0, i<n$, and $p$ is a prime such that $2 \leq p \leq n-1, n \neq 0(\bmod p)$, then $p$ divides $\chi(M)$. Both theorems imply that an almost-complex homology sphere must have dimension less than or equal to 6 . (See [5, p. 278] or [1].) Theorems 2 and 3 improve theorems of Gilmore [3] who used the obstruction theory techniques of Mahowald [6]. Theorem 2 was proven in [3] with the additional hypothesis that $H_{2}(M ; Z)$ is torsion free and Theorem 3 was proven with the assumptions that $\mathrm{H}_{2}(M ; \mathbf{Z}), \mathrm{H}_{4}(M ; \mathrm{Z})$ and $\mathrm{H}_{6}(M ; \mathrm{Z})$ are torsion free.

2. The obstruction formulas. If $y^{n}$ is in $H^{n}(Y ; G)$, let $y_{\#}^{n}: \pi_{n}(Y) \rightarrow G$ be the composite of the Hurewicz homomorphism and evaluation. Proofs of the next two formulas are in [7]. If $f, g: X \rightarrow Y$ are two maps and the homotopy obstruction $\mathcal{O}^{n}(f, g)$ is nonvoid, then

$$
y_{*}^{n} \Theta n(f, g)=f^{*} y^{n}-g^{*} y^{n} .
$$

If $F \rightarrow Y \rightarrow B$ is a fibration with fiber $F, a^{n-1}$ in $H^{n-1}(F ; G)$ is a class transgressing to $b^{n}$ in $H^{n}(B ; G)$, and $f: X \rightarrow B$ a map such that the lifting obstruction is nonvoid, then

$$
-a_{*}^{n-1} \Theta^{n}(f)=f^{*} b^{n}
$$

3. The proof of Theorem 1. Let $f: M \rightarrow B U(n)$ represent the almostcomplex structure of $M$. If $*$ is the constant map and $\mathcal{O}^{2 i}(f, *)$ is nonvoid, it follows from Bott's computation of the stable Hurewicz homomorphism of $B U(n)$ (see $\left[5\right.$, p. 278] or $\left[7\right.$, p. 95]) and (2.1) that in $H^{2 i}(M ; Z)$ we have $(i-1) ! \mathcal{O}^{2 i}(f, *)=c_{i}(M)$. It now follows from the hypotheses that $(n-1) ! \cdot$

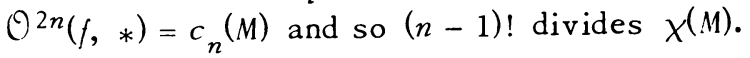

4. The proofs of Theorems 2 and 3 . If $M$ is an almost-complex manifold, let $f: M \rightarrow B U(n)$ represent its complex tangent bundle. It is clear that complex $\operatorname{span}(M) \geq q$ if and only if the map $f$ lifts to the total space of the fibration $W_{n, q} \rightarrow B U(n-q) \rightarrow B U(n)$, where $W_{n, q}$ is the Stiefel variety of complex $q$-frames in complex $n$-space. It is well known that $H^{*}\left(W_{n, q} ; \mathbf{Z}\right)$ is an exterior algebra with generators $a_{i}$ in $H^{2 i+1}\left(W_{n, q} ; \mathbf{Z}\right)$, $n-q \leq i \leq n-1$. (See [2, p. 444].) It is easy to see that $a_{i}$ transgresses to the Chern class $c(i+1)$. It follows immediately from (2.2) that $-a_{i} \mathcal{O}^{2(i+1)}(f)=c_{i+1}(M)$. If $\pi_{2 i+1}\left(W_{n, q}\right)$ is isomorphic to $\mathrm{Z}, a_{i \#}$ will be 
multiplication by an integer, and the assumption $c_{i+1}(M)=0$ will imply that $\mathcal{O}^{2(i+1)}(f)=0$ if suitable assumptions are made about the torsion subgroup of $H^{2(i+1)}(M ; Z)$.

The groups $\pi_{2 i+1}\left(W_{n, q}\right)$ are periodically isomorphic to $\mathbf{Z}$ if $n$ is large, $q=4$ or 5 , and $n-q$ is odd. The table below shows the homotopy groups and the Hurewicz homomorphism for $W_{n, 4^{*}}$ The homotopy groups are taken from [3]. The space $W_{n, q}$ is $2(n-q)$-connected. The Hurewicz homomorphism can be computed using a routine inductive argument based on the fibration $W_{n-1, q-1} \rightarrow W_{n, q} \rightarrow S^{2 n-1}$. Since $n$ is large, the homology spectral sequence of this fibration collapses to a terminating exact sequence which we may use with the homotopy sequence in the inductive argument. The argument begins with the fibration $S^{2 n-3} \rightarrow W_{n, 2} \rightarrow S^{2 n-1}$ which can be used with the tables in [3] to show that $b: \pi_{2 n-3}\left(W_{n, 2}\right) \rightarrow H_{2 n-3}\left(W_{n, 2}\right)$ is multiplication by 2 if $n$ is odd. It is easy to use the fiberings over odd dimensional spheres to successively compute the Hurewicz homomorphism for $W_{n, q}, q=3,4,5$ and $n-q$ odd. In certain dimensions, the exact value of the group or of $b(1)$ depends on the congruence classes of $n$ modulo 3 and 8 . These values are not important in computing obstructions.

$$
W_{n, 4}, n \text { odd and } n \geq 9
$$

\begin{tabular}{|c|c|c|}
\multicolumn{2}{c}{$\pi_{i}\left(W_{n, 4}\right)$} & $b(1)$ \\
\hline $2 n-7$ & $\mathbf{Z}$ & 1 \\
$2 n-6$ & 0 & 0 \\
$2 n-5$ & $\mathbf{Z}$ & 2 \\
$2 n-4$ & 2 or 3-torsion group & 0 \\
$2 n-3$ & $\mathrm{Z}$ & $1,2,3,4,6,12$ \\
$2 n-2$ & 2 or 3-torsion group & 0 \\
$2 n-1$ & $\mathbf{Z}$ & $3,6,8,12$ \\
\hline
\end{tabular}

If $n$ is even and $n \geq 8$, the table of homotopy groups of $W_{n, 5}$ looks exactly the same as the above table with the dimensions running from $2 n-9$ to $2 n-3$. If $n \neq 0(\bmod 8)$, it is shown in [4] that $\pi_{2 n-1}\left(W_{n, 5}\right)$ is isomorphic to $\mathrm{Z}$ and since $n \geq 10$, it follows from the Hurewic $z$ isomorphism theore $\mathrm{m}$ modulo finite abe lian groups that $b: \pi_{2 n-1}\left(W_{n, 5}\right) \rightarrow H_{2 n-1}\left(W_{n, 5}\right)$ is not zero.

Theorems 2 and 3 now follow easily, if the hypotheses on the homology 
groups are translated into terms of cohomology via Poincaré duality. In the case of Theorem 1, it is easy to see that the hypotheses imply that $m \Theta^{2(n-1)}(f)=c_{n-1}(M)$, where $m$ is a divisor of 12. The assumption $c_{n-1}(M)=0$ is enough to imply that $\mathcal{O}^{2(n-1)}(f)$ is zero because $H^{2(n-1)}(M ; Z)$ cannot have any 2 or 3 -torsion elements since we assume that $H^{2 n-3}\left(M ; \mathrm{Z}_{p}\right)=0$ for $p=2$ or 3 , and the $p$-torsion subgroup of $H^{2(n-1)}(M ; Z)$ is the image of the Bockstein $\delta: H^{2 n-3}\left(M ; Z_{p}\right) \rightarrow$ $H^{2(n-1)}(M ; Z)$. The next obstruction is zero because $M$ is simply connected and the hypothesis $c_{n}(M)=0$ implies that the final obstruction is zero because $b(1) \neq 0$ and $M$ is orientable. Theorem 3 is proved in the same way.

\section{REFERENCES}

1. E. C. Boes, Solution of the almost-complex spheres problem using K-theory, Rocky Mountain J. Math. 1 (1973), 19-22.

2. A. Borel and J.-P. Serre, Groupes de Lie et puissances réduites de Steenrod, Amer. J. Math. 75 (1953), 409-448. MR 15, 338.

3. M. Gilmore, Complex Stiefel manifolds, some homotopy groups and vector fields, Bull. Amer. Math. Soc. 73 (1967), 630-633. MR 35 \#419.

4. - Homotopy groups of complex Stiefel manifolds, Ph. D. Thesis, Univ. of California, Berkeley, 1967.

5. D. Husemoller, Fibre bundles, McGraw-Hill, New York, 1966. MR 37\#4821.

6. M. Mahowald, On obstruction theory in orientable fibre bundles, Trans. Amer. Math. Soc. 110 (1964), 315-349. MR 28 \#620.

7. P. Olum, Factorizations and induced homomorphisms, Advances in Math. 3 (1969), 72-100. MR 38 \#6590.

DEPARTMENT OF MATHEMATICS, UNIVERSITY OF HAWAII, HONOLULU, HAWAII 96822 